Thinking and Being 



\section{Thinking and Being}

IR AD KIMHI 
Copyright (@) 2018 by the President and Fellows of Harvard College All rights reserved

Printed in the United States of America

First printing

Library of Congress Cataloging-in-Publication Data

Names: Kimhi, Irad, author.

Title: Thinking and being / Irad Kimhi.

Description: Cambridge, Massachusetts : Harvard University Press, 2018. | Includes bibliographical references and index.

Identifiers: LCCN 2017049673 | ISBN 9780674967892 (cloth : alk. paper)

Subjects: LCSH: Frege, Gottlob, 1848-1925. | Analysis (Philosophy) | Thought and thinking. | Logic. | Ontology. | Language and logic. Classification: LCC B808.5 .K56 2018 | DDC 128 / .33—dc23

LC record available at https://lccn.loc.gov/2017049673.

Jacket design: Lisa Roberts

Jacket art: (detail) Bedroom with Photo (2005) by Diti Almog, $9 \times 18$ " | acrylic on aircraft plywood 